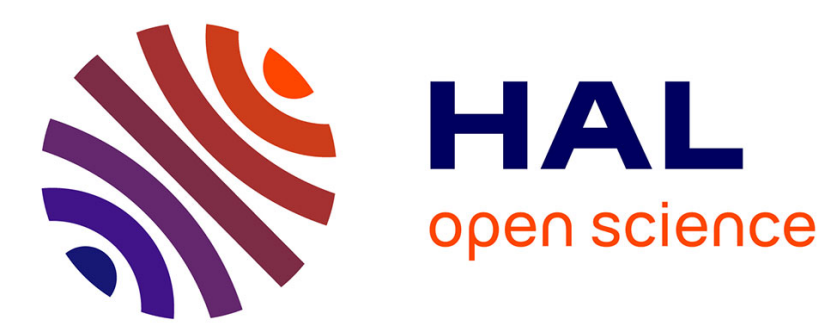

\title{
Controlling wet adhesion with elasticity
}

Camille Duprat, Camille Noûs, Suzie Protière

\section{To cite this version:}

Camille Duprat, Camille Noûs, Suzie Protière. Controlling wet adhesion with elasticity. Soft Matter, 2020, 10.1039/D0SM00618A . hal-03022064

\section{HAL Id: hal-03022064 \\ https://hal.science/hal-03022064}

Submitted on 10 Dec 2020

HAL is a multi-disciplinary open access archive for the deposit and dissemination of scientific research documents, whether they are published or not. The documents may come from teaching and research institutions in France or abroad, or from public or private research centers.
L'archive ouverte pluridisciplinaire HAL, est destinée au dépôt et à la diffusion de documents scientifiques de niveau recherche, publiés ou non, émanant des établissements d'enseignement et de recherche français ou étrangers, des laboratoires publics ou privés. 


\title{
Controlling wet adhesion with elasticity ${ }^{\dagger}$
}

\begin{abstract}
Camille Duprat ${ }^{a}$, Camille Noûs ${ }^{b}$ and Suzie Protière ${ }^{c}$
We consider the wet adhesion between two deformable fibers. We show that a strong adhesive force can be maintained by coupling the fibers deformation with capillarity. We further identify a regime where, contrary to capillary adhesion, the pull-off force remains constant throughout debonding. In this peeling regime, elasticity, which tends to minimize the deformation of the object, is balanced by capillarity which maximizes the liquid spreading. We show that the adhesive force and the existence region of this self-adjusted peeling regime depend on a single dimensionless parameter, and can thus be controlled by tuning the material properties.
\end{abstract}

\section{Introduction}

The adhesion between two soft solids is strongly affected by the presence of a small amount of liquid. For example, capillarity controls the bioadhesion of insects tarsi $1+4 \sqrt{4}$ and condensation can modify the propagation of a crack tip $\left.{ }^{5}+7\right]$ or affect the measurement with an AFM probe ${ }^{899}$. Conversely, elastic deformation affects the wet adhesion of soft materials ${ }^{10 \mid 11}$, and in particular fibers ${ }^{12113}$. Determining the exact force balance characterizing this type of adhesion requires to link the spreading of the liquid, the deformation of the solid and the adhesive force. Previous efforts to characterize these interactions have focussed on the adhesion of deformable spheres and substrates, extending the Hertz theory of contact to account for the effect of a capillary meniscus 11114 , or on the spreading induced by the soft solid deformation $15 \mid 16$. In this paper, we directly measure the forces generated when two deformable slender objects are bonded by a finite volume of liquid. We find a regime where the adhesion force remains stable as the substrates are pulled apart. Moreover this force can be controlled by adjusting the tension or varying the material's properties. This behavior is then compared to the peeling test classically studied when removing an adhesive tape.

\section{Experiments}

As a model system, we consider two fibres of radius $r$ clamped at both ends and wetted by a droplet of a perfectly wetting liquid (figure 1 a). The effective modulus of the fibres can be adjusted by controlling the tension within the fibres, i.e. imposing a prestrain $\varepsilon$, to span a large range of flexibilities, from rigid-like to soft and deformable. The deformability, i.e. the amount of deformation that can be sustained by capillary forces, depends on

\footnotetext{
${ }^{a}$ LadHyX, CNRS, École Polytechnique, Institut Polytechnique de Paris, UMR 7646, 91128 Palaiseau, France, E-mail: camille.duprat@ladhyx.polytechnique.fr

${ }^{b}$ Cogitamus laboratory, 1 3/4 rue Descartes, 75005 Paris, France

${ }^{c}$ Institut Jean Le Rond d'Alembert, CNRS, Sorbonne Université, UMR 7190, F-75005 Paris, France, E-mail: protiere@ida.upmc.fr

$\dagger$ Electronic Supplementary Information (ESI) available: [details of any supplementary information available should be included here].
}

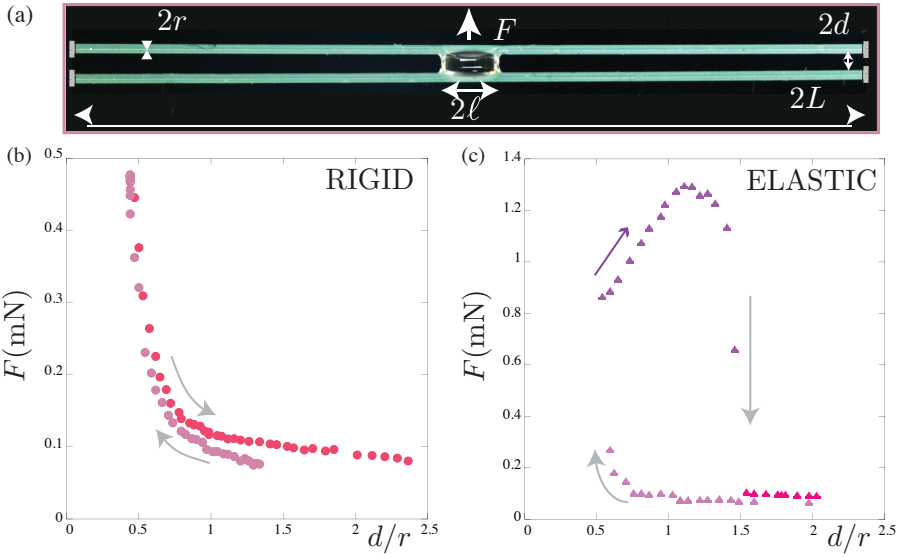

Fig. 1 (a) Schematic of the experiment with the initial configuration of a drop placed between two elastic fibres. Force exerted by the drop (b) for rigid nylon fibres $\left(\mathscr{N}_{c s} \gg 1\right.$, circles), and (c) for flexible elastomeric fibres $\left(\mathscr{N}_{c s}=2769\right.$, triangles $)$.

the material and geometrical properties (size $r$, effective elasticity $E \varepsilon$, liquid surface tension $\gamma$ ), and is quantified by comparing tension $\left(T \equiv E \varepsilon r^{2}\right)$ and capillary force $(F \equiv \gamma r)$ to define a dimensionless capillary-stretching $\frac{17118}{n}$ number $\mathscr{N}_{c s}=E \varepsilon r / \gamma$. Rigid fibres $\left(\mathscr{N}_{c s} \gg 1\right)$ are obtained using nylon fibres of radius $r=0.2 \mathrm{~mm}$ under large pre-strain. Flexible fibers are obtained by moulding an elastomer (Polyvinyl Siloxane (PVS), Zhermack elite double) in capillary tubes of radii $r=0.2,0.3$ and $0.4 \mathrm{~mm}$ with various Young moduli $E=0.4,0.5$ and $0.9 \mathrm{MPa}$. The fibres are then attached to a laser-cutted rigid structure with a pre-strain $1 \%<\varepsilon<30 \%$. We used droplets of mineral oil ( $\gamma=30 \mathrm{mN} / \mathrm{m}$, contact angle $\theta \approx 0^{\circ}$, no contact angle hysteresis) with a fixed volume $V=3 \mu \mathrm{L}$. The top fibre is attached to a micro-sensor (Lauda Scientific tensiometer, precision $10 \mu \mathrm{N}$ ) while the bottom one is attached to a microcontrolled platform on which we perform steps of $15 \mu \mathrm{m}$. We measure the adhesive force $F$ exerted by the liquid on the fibre as we increase or decrease the interfibre distance $d$ incrementally. At each step, we wait for equilibrium to be reached (stable force 


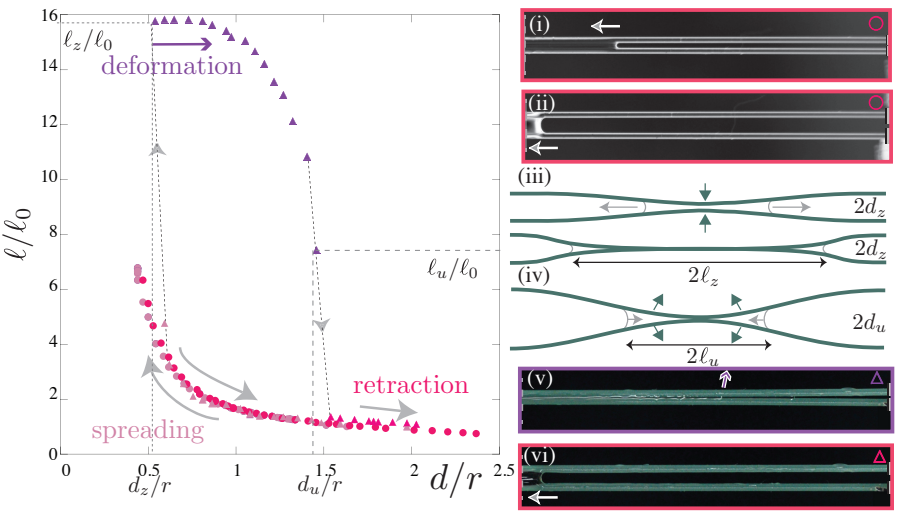

Fig. 2 Evolution of the wetted length when varying the inter-fibre distance for rigid (circles) and flexible fibres (triangles), with the same parameters as Fig. 1 As the fibre are pulled apart, we observe two different scenarii as shown in (i)-(iv): liquid retraction (grey arrows) and fibre deformation (purple arrows). Colors and symbols match the different configurations throughout the figures.

and spreading length), typically obtained after 1 minute for short capillary bridge and up to 30 minutes for zipped liquid columns, to ensure that the interfibre distance is changed quasi-statically and no dynamical effects are present.

For rigid fibres, the force increases rapidly with decreasing distance as the liquid bridge spreads (figure $1 \mathrm{~b}$ ), and decreases as the fibres are pulled apart and the liquid retracts in a reversible manner, which is typical of capillary adhesion. We now proceed to the same experiment with slightly more elastic fibres of same radius, wetted by the same liquid (figure $1 \mathrm{c}$ ). Starting from the same initial condition, the force first increases in a similar fashion, until a critical interfibre distance at which we observe a jump in the force. As the fibres are pulled apart, the force keeps on increasing until it reaches a maximum, after which it decreases sharply. We can link the variations in these measured adhesive forces with changes in the liquid/fibre system morphology, whose evolution is monitored by measuring the wetted length $2 \ell$, normalized by the initial length of the liquid bridge $2 \ell_{0}$ (figure 2). Starting from a drop, the liquid between the fibres remains as a spreading liquid bridge as we decrease $d$ and the evolution of the liquid length is identical for both rigid and elastic fibres. However, for elastic fibres, the liquid spreads spontaneously at a critical interfibre distance $d_{z}$, resulting in the formation of a zipped state $e^{17}$ where the fibres are parallel and almost completely in contact at the center with a spreading length $2 \ell_{z}$, with a deformation confined to the edges where the menisci are formed (figure 2(iii)). We note that in this zipped state the fibers are not in contact as a small amount of liquid is always present between the fibers. As the fibres are pulled apart, two different scenarii are observed: for rigid fibres, the liquid simply retracts without hysteresis (figure 2(i-ii)), whereas for flexible fibres there is first a small region where the wetted length is constant and the fibres are deformed (purple arrows, figure2(v)) before the length decreases to jump back to the initially observed liquid bridge, i.e. unzip, at a critical distance $d_{u}$ corresponding to a wetted length $2 \ell_{u}$ (figure 2 (iv-vi)).

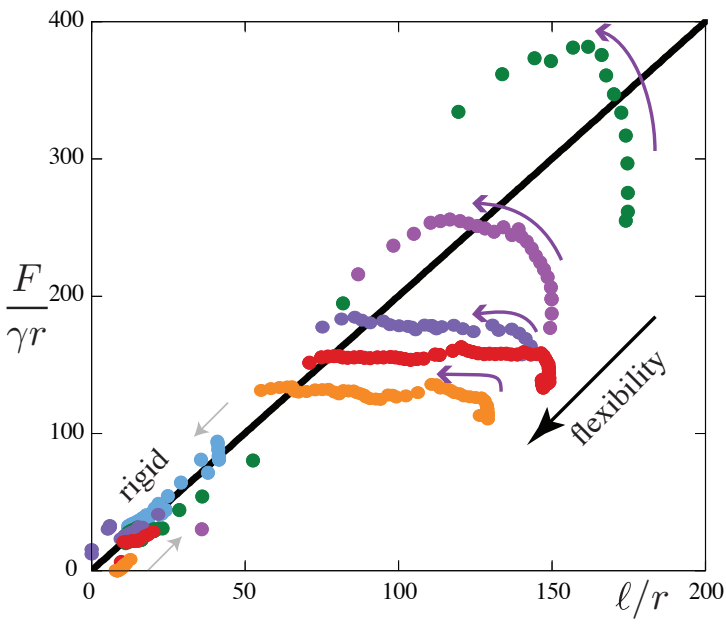

Fig. 3 Evolution of the force normalized by the characteristic capillary force $\gamma r$ as a function of the spreading length $\ell / r$ throughout a bonding and debonding cycle. Experimental data for rigid fibres (light blue), $\mathscr{N}_{c s}=$ 2769 (green), $\mathscr{N}_{c s}=2077$ (light purple), $\mathscr{N}_{c s}=1500$ (purple), $\mathscr{N}_{c s}=750$ (red), $\mathscr{N}_{c s}=517$ (orange). Solid line: force exerted by a capillary bridge $F=3.2 \gamma \ell$. Rigid fibres follow this behaviour (grey arrows) while elastic fibres depart from this behaviour as they deform (purple arrows).

\section{From wetting to peeling}

For rigid fibres, the force is directly linked to the wetted length: as the liquid spreads or retracts, the force increases or decreases. However, for flexible fibres, the force depends on both the wetted length and the fibre deformation. The jump in the force is associated with the "zipping" transition as the liquid spreads between the fibres. As we pull on the fibres, we first increase their deformation with a constant wetted length and the force must increase, until the liquid starts to retract and the force decreases. The maximum in the force is thus due to a combined elasto-capillary effect. We plot the force as a function of the wetted length (figure 3) for different fibre elasticity. For rigid fibres, the force is proportional to the length (blue points), as expected from a capillary bridge. We found here that $F=1.6 \gamma 2 \ell$. The prefactor 1.6 is close to 2 , as expected for a liquid bridge, but depends on the complex threedimensional shape of the drop and is thus dependent on the drop volume; in fact, the force decreases as the volume increases 19220 . However, a complete characterization of this force is beyond the scope of this study and we thus keep the volume constant here. The force can be increased by bringing the fibres close together. With elastic fibres, the deformation induces a spontaneous maximal spreading and thus a strong increase of the force. However, this force is smaller than the force that would be obtained in the rigid case for the same wetted length. The force increases as the length remains constant and then reaches a maximum. As we increase the fibre flexibility, this maximum force decreases. Moreover, for even more flexible fibres, instead of observing a maximum in the force we observe a plateau; the force is constant for a large range of lengths that increases with flexibility.

In this plateau regime, we then plot the corresponding force and length in figure $4 \mathrm{a}-\mathrm{b}$. We also report the value of the angle $\theta$ between the deformed fibres, as a measure of the deformation (figure (4b-c). In addition to the spreading, retraction and deformation 

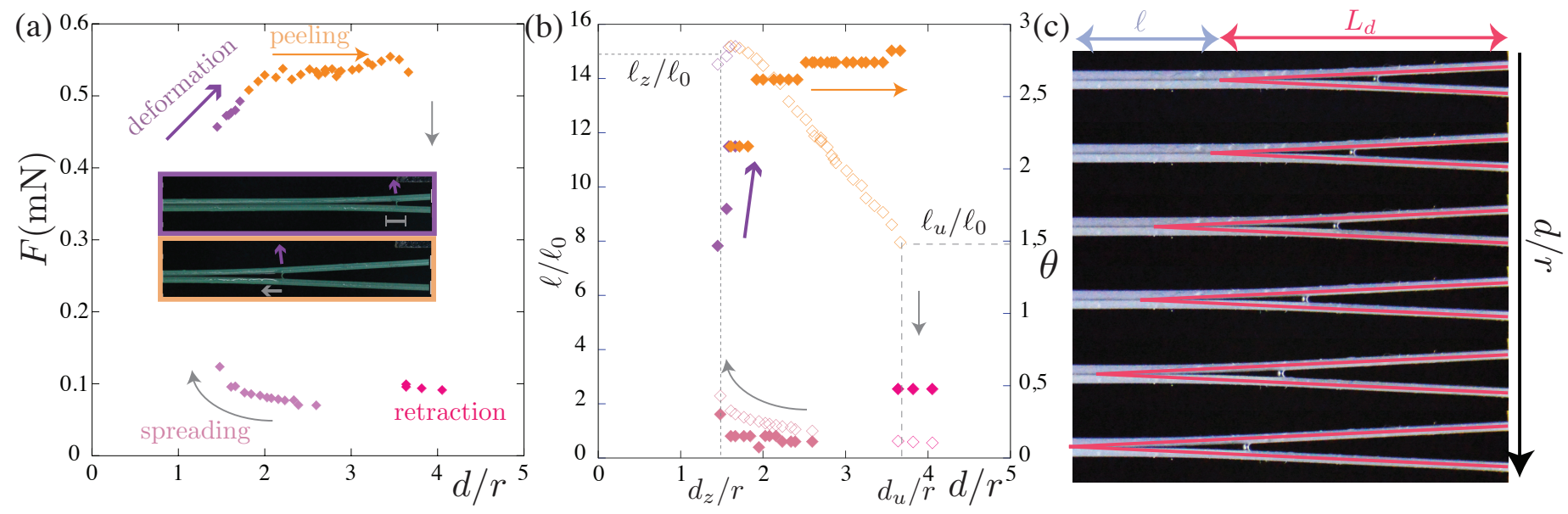

Fig. 4 Evolution of (a) the force, (b) the wet length (open symbols, left axis) and angle (filled symbols, right axis) between the fibers $\theta$ for deformable fibers $\left(\mathscr{N}_{c s}=461\right)$. (c) Images of the deformed fibres as they are pulled apart, highlighting the constant angle (in red).

states observed previously, we have an additional "peeling" state during debonding: the fibres deform and the liquid retracts simultaneously (orange points in figure 4). The fibres are parallel along a certain distance which decreases linearly when $d$ increases (figure 4p). The deformation of the fibres is confined to the meniscus region where the fibres form a constant angle $\theta$ (figure $4 \mathrm{c}$ ) reminiscent of the peeling of an adhesive. While in the classical peeling test ${ }^{21}$ the force depends on the prescribed angle, here with wet elastic fibres the deformation self-adjusts with the decrease in wet length, thus leading to a constant intrinsic angle and a resulting force plateau.

In this peeling regime, the shape of the fibres is thus given by a balance between elasticity that minimizes the deformation, i.e. minimizes the dry length $L_{d}=L-\ell$, and capillarity that maximizes spreading of the liquid, i.e. the wetted length $2 \ell$ (figure $4 \mathrm{c}$ ). We assume a simplified model for the system where the capillary force is generated by a liquid column in the coalesced part of the fibres of length $2 \ell$, i.e we neglect the effect of the liquid stored in the menisci at the edges of the column. The elastocapillary balance is then obtained by minimizing the total energy of the system $\mathscr{E}=$ $E \varepsilon \pi r^{2} d^{2} / L_{d}-2 \gamma r \ell$, giving the dry length

$$
L_{d}=c_{0} d \sqrt{\mathscr{N}_{c s}}
$$

where the constant $c_{0} \simeq 1.25$ has been determined previously 17 . As we pull on the fibre, we increase the distance $d$ and the dry length $L_{d}$ increases, i.e. the liquid retracts as the deformation increases. The length of liquid decreases linearly with the distance $d$ as observed in figure $4 \mathrm{~b}$. This equilibrium implies that the angle between the fibres remains constant as seen in Fig. $4 \mathrm{c}$. Since the angle is always small, it is given by

$$
\theta \simeq \frac{d}{L_{d}} \sim \frac{1}{\sqrt{\mathscr{N}_{c s}}}
$$

The peeling process is represented in the inset of Fig. 5 . The pulling force is given by the balance between the work necessary to displace the fibre $F_{T} \delta(1-\cos \theta)$ and the adhesion $\alpha \gamma r \delta$ ( $\alpha$ depends on the complex shape of the liquid column between the fibres and of the menisci), i.e. the force only depends on the peeling angle $\theta$. The vertical force is thus given by

$$
F=F_{T} \theta=\frac{\alpha \gamma r}{2 \theta}=c_{1} \gamma r \sqrt{\mathscr{N}_{c s}}
$$

which is independent of the distance $d$ or the liquid length $\ell$. We measure the maximum force for fibres of different radii and moduli, i.e. several $\mathscr{N}_{c s}$ and aspect ratios, as reported in figure 5 a. The constant force in the "peeling" region only depends on the material properties $\mathscr{N}_{c s}$, in good agreement with equation (3) with a constant $c_{1}=1.6$.

As $\mathscr{N}_{c s}$ decreases, the length of the plateau region increases, i.e. the fibres are in the constant force peeling region for a wider range of distances (Fig. 3). The length of the plateau can be estimated by comparing the maximum spread length at zipping $2 \ell_{z}$ and the length at unzipping $2 \ell_{u}$. Zipping occurs when the distance between the fibres reaches a critical distance $d_{z}$ at which spreading is favored. In the case of fibres, we found $d_{z}=0.57 r$ [17. In general, the zipped length can be expressed as

$$
2 \ell_{z}=2\left(L-L_{d}\left(d=d_{z}\right)\right)=2\left(L-d_{z} \sqrt{\mathscr{N}_{c s}}\right) .
$$

As the distance between the fibres increases, the dry length increases until reaching the center of the fibres $\left(L_{d} \sim L\right)$, leading to the unzipping of the fibres at a distance $d_{u}$. We measure the critical unzipping as $L_{d}\left(d=d_{u}\right)=c_{2} L$, where $c_{2} \leq 1$ and we find

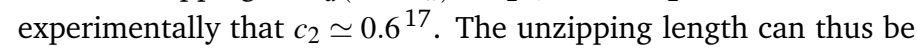
estimated as

$$
2 \ell_{u}=2\left(L-L_{d}\left(d=d_{u}\right)\right)=2 L(1-c 2) .
$$

The plateau length $2 \ell_{p}=2\left(\ell_{z}-\ell_{u}\right)$ can then be written as

$$
\frac{\ell_{p}}{L}=c_{2}-c_{3} \sqrt{\mathscr{N}_{c s}}
$$

where $c_{3}=\frac{d_{z}}{L}$. We measure the extent of the plateau region for several $\mathscr{N}_{c s}$ and aspect ratios. The expression (6) is in good agreement with experimental data (figure $5 \mathrm{~b}$ ). For highly flexible fibres $\left(\mathscr{N}_{C S} \ll 1\right)$, the plateau length is close to $L$, i.e. peeling is 

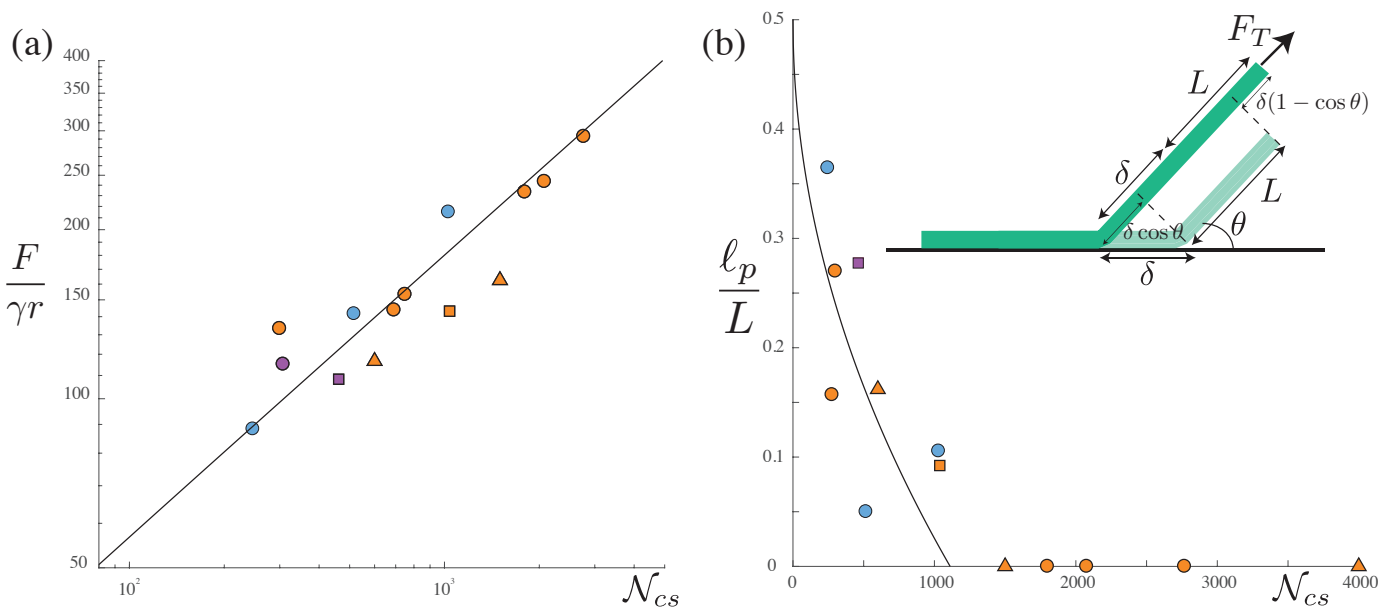

Fig. 5 (a) Measurement of the maximum adhesion strengths for various fibre-liquid systems as a function of the capillary-stretching number $N_{c s}$. Line: $F / \gamma r=1.6 \sqrt{\mathscr{N}_{c s}}$. (b) Peeling length as a function of $N_{c s}$ and aspect ratio. Fibres of lengths $L=40 \mathrm{~mm}$ and radius $r=0.2 \mathrm{~mm}$ (circles), $r=0.3 \mathrm{~mm}$ (square), $r=0.4 \mathrm{~mm}$ (triangle) and modulus $E=0.9 \mathrm{MPa}$ (yellow), $E=0.5 \mathrm{MPa}$ (blue), $E=0.4 \mathrm{MPa}$ (pink), and various tensions. Inset: sketch of the peeling process. Line: eq. 6 with $c_{2}=0.5$ and $c_{3}=0.011$, in agreement with the expected range of values.

always favored. Increasing $\mathscr{N}_{c s}$, i.e. increasing the rigidity of the fibres, reduces the length of the plateau until a critical value $\ell_{p}=0$ at which no peeling occurs. The critical peeling value, given by $\mathscr{N}_{c s}=\left(\frac{c 2}{c 3}\right)^{2}$, strongly depends on the aspect ratio $r / L$ through the critical distance at zipping. We have performed preliminary experiments on thin (600 microns) and narrow $(1.5 \mathrm{~mm}$ ) plates and obtained qualitatively similar results, with a zipping transition and a force plateau. It would be interesting to extend our results to other geometries, in particular to investigate situations where three-dimensional effects will occur, as will be the case for larger plates where the liquid takes the form of a cylindrical capillary bridge rather than a drop, i.e. does not wet the entire width of the plate.

\section{Conclusions}

Here we have shown that the adhesive force between wet flexible objects reaches a plateau with a maximum value and a range that are completely determined by a single parameter $\mathscr{N}_{c s}$. It is thus possible to control both the value of the force and the extent of the plateau by dynamically adjusting the tension within the fibres, or choosing the desired material properties. While large forces could be reached with rigid objects, it requires to bring them in close contact as the force increases linearly with the spreading length, which is difficult to obtain due to alignement or roughness. With flexible slender objects, large adhesion forces can be reached more easily as flexibility favors spreading, even for large distances. In addition, in rigid wet adhesion, a small variation in gap size induces larges variations in the adhesive force; with the self-adjusting peeling, the force can be kept stable for a larger range of distances. Furthermore, for highly flexible objects, i.e. at small $\mathscr{N}_{c s}$, the unzipping distance $d_{u}$ can reach large values, and in particular distances which are too large to maintain a capillary bridge between the fibres after unbonding 17; through zipping and the self-adjusting peeling, flexibility thus extends the adhesion to inter-fiber distances impossible to reach with rigid fibers. These findings open new routes towards the design and optimization of novel materials or processes using wet adhesion 22,25 .

\section{Conflicts of interest}

There are no conflicts to declare.

\section{Acknowledgements}

We thank Benoit Roman for helpful discussions about peeling, and Quentin Magdelaine and Nsemi Nsimba for help with the experiments.

\section{Notes and references}

1 J.-H. Dirks and W. Federle, Soft Matter, 2011, 7, 11047.

2 A. E. Kovalev, M. Varenberg and S. N. Gorb, Soft Matter, 2012, 8, 7560 .

3 L. Xue, A. Kovalev, A. Eichler-Volf, M. Steinhart and S. N. Gorb, Nat Commun, 2015, 6, 6621.

4 T. Gilet, S.-M. Gernay, L. Aquilante, M. Mastrangeli and P. Lambert, Soft matter, 2019, 15, 3999-4007.

5 A. Grimaldi, M. George, G. Pallares, C. Marliâlíre and M. Ciccotti, Phys. Rev. Lett., 2008, 100, 165505.

6 G. Pallares, A. Grimaldi, M. George, L. Ponson and M. Ciccotti, Journal of the American Ceramic Society, 2011, 94, 2613-2618.

7 E. Charlaix and M. Ciccotti, Handbook of nanophysics, CRC press, 2010.

8 A. A. Feiler, J. Stiernstedt, K. Theander, P. Jenkins and M. W. Rutland, Langmuir, 2007, 23, 517-522.

9 M. S. Rodrigues, L. Costa, J. Chevrier and F. Comin, Appl. Phys. Lett., 2012, 101, 203105.

10 K. Li and S. Cai, Soft matter, 2014, 10, 8202-8209.

11 H.-J. Butt, W. J. P. Barnes, A. del Campo, M. Kappl and F. SchÃűnfeld, Soft Matter, 2010, 6, 5930. 
12 B. N. J. Persson, C. Ganser, F. Schmied, C. Teichert, R. Schennach, E. Gilli and U. Hirn, J. Phys.: Condens. Matter, 2013, 25, 045002.

13 M. Soleimani, R. J. Hill and T. G. van de Ven, Langmuir, 2015, 31, 8328-8334.

14 M. Zakerin, M. Kappl, E. H. G. Backus, H.-J. Butt and F. SchÃünfeld, Soft Matter, 2013, 9, 4534.

15 J. S. Wexler, T. M. Heard and H. A. Stone, Phys. Rev. Lett., 2014, 112, 066102.

16 M. Butler, F. Box, T. Robert and D. Vella, Phys. Rev. Fluids, 2019, 4, 033601.

17 C. Duprat and S. Protiere, EPL (Europhysics Letters), 2015, 111, 56006.

18 J. Bico, É. Reyssat and B. Roman, Annual Review of Fluid Mechanics, 2018, 50, 629-659.
19 C. Duprat, S. Protiere, A. Y. Beebe and H. A. Stone, Nature, 2012, 482, 510-513.

20 P.-B. Bintein, PhD thesis, Universite Pierre et Marie Curie, 2015.

21 K. Kendall, J. Phys. D: Appl. Phys., 1975, 8, 1449-1452.

22 S. N. Gorb, Philosophical Transactions of the Royal Society A: Mathematical, Physical and Engineering Sciences, 2008, 366, 1557-1574.

23 S. Baik, D. W. Kim, Y. Park, T.-J. Lee, S. Ho Bhang and C. Pang, Nature, 2017, 546, 396-400.

24 P. Van Nguyen, N. Van Huynh, T. T. Phan et al., 2018 IEEE International Conference on Soft Robotics (RoboSoft), 2018, pp. 418-423.

25 F. Meng, Q. Liu, X. Wang, D. Tan, L. Xue and W. J. P. Barnes, Phil. Trans. R. Soc. A, 2019, 377, 20190131. 